\title{
A non-auditory measure of interference predicts distraction by competing speech in older adults
}

\author{
Esther Janse ${ }^{1,2}$ \\ ${ }^{1}$ Utrecht institute of Linguistics, Utrecht University, Utrecht, The Netherlands \\ ${ }^{2}$ Max Planck Institute for Psycholinguistics, Nijmegen, The Netherlands
}

\begin{abstract}
In this study, older adults monitored for pre-assigned target sounds in a target talker's speech in a quiet (no noise) condition and in a condition with competing-talker noise. The question was to which extent the impact of the competing-talker noise on performance could be predicted from individual hearing loss and from a cognitive measure of inhibitory abilities, i.e., a measure of Stroop interference. The results showed that the non-auditory measure of Stroop interference predicted the impact of distraction on performance, over and above the effect of hearing loss. This suggests that individual differences in inhibitory abilities among older adults relate to susceptibility to distracting speech.
\end{abstract}

Keywords: Speech perception; Aging; Speech in noise; Individual differences: Inhibitory control; Informational masking.

Listening to one person speaking while there are other talkers present in the background can be a challenging task, particularly for older listeners. The primary reason for older adults being more affected by competing speech than younger adults is age-related hearing loss. Yet, older adults tend to have greater difficulty understanding speech in noisy backgrounds than young adults even when the age groups are matched for pure-tone hearing acuity (Dubno, Dirks, \& Morgan, 1984). This could be due to two additional age-related issues. First, age groups may differ in temporal and frequency

This research was supported by an Innovational Research Incentive Scheme Veni grant from the Netherlands Organization for Scientific Research to the author (Project number 275-75-004).

Address correspondence to: Esther Janse, CLSM, P.O. Box 310, 6500 AH Nijmegen. The Netherlands. E-mail: esther.janse@mpi.nl 
selectivity (Gordon-Salant \& Fitzgibbons, 1993; Phillips, Gordon-Salant, Fitzgibbons, \& Yeni-Komshian, 2000; Tremblay, Piskosz, \& Souza, 2003). Second, there are indications that age-related cognitive decline, e.g., reduced attentional resources in older adults (Anderson, Craik, \& Naveh-Benjamin, 1998), may play a role as well (Salthouse, 1991; Tun, McCoy, \& Wingfield, 2009). Decreases in the ability to focus and maintain attention would be particularly harmful for speech understanding in listening conditions with multiple talkers present.

Hasher and Zacks (1988) formulated the inhibitory deficit hypothesis of aging. According to Hasher and Zacks, inhibition prevents goal-irrelevant information from interfering with working memory and taking up resources that would otherwise be free for processing of task- or goal-relevant information (cf. also Connelly, Hasher, \& Zacks, 1991; Hasher, Zacks, \& May, 1998; Head, Kennedy, Rodrigue, \& Raz, 2009; Zacks \& Hasher, 1994).

Age differences suggestive of decreased inhibitory control have been found with different inhibitory-control measures, such as tests of Stroop interference (e.g., Bugg, DeLosh, Davalos, \& Davis, 2007; Houx, Jolles, \& Vreeling, 1993; Kwong See \& Ryan, 1995; Spieler, Balota, \& Faust, 1996), and Wisconsin Card sorting tests (cf. for a review, Rhodes, 2004).

Impaired inhibitory control may relate to problems with speech perception in multi-talker settings, as the informational content of the competing speech may make it particularly challenging to focus on the target talker and to effectively inhibit the distractor talker (cf. e.g., Brungart, 2001, for a review, on the distinction between informational and energetic masking, with energetic masking referring to reduced intelligibility of the target signal due to acoustic overlap between target and masker, and informational masking referring to the situation in which the masker not only makes the target signal less intelligible, but may also compete for the listener's attention). For both young and older listeners, masking by a multi-talker babble in a known language has been shown to be more deleterious to target speech recognition than masking by multi-talker babble in an unknown language (Garcia Lecumberri \& Cooke, 2006; Van Engen \& Bradlow, 2007). Aging has been shown to increase the language-familiarity effect for informational masking: Tun, O'Kane, and Wingfield (2002) found that older adults were disproportionally affected by competing speech spoken in their native language, relative to young adults.

Thus, cognitive factors beyond age-related peripheral hearing impairment and central auditory processing may play a role in older adults' problems with competing speech in the background (but see e.g., Guerreiro, Murphy, \& van Gerven, 2010; Murphy, McDowd, \& Wilcox, 1999; Schneider, Daneman, Murphy, \& Kwong See, 2000). More direct evidence that cognitive abilities relate to listening performance in conditions with competing speech comes from studies that take an individual-differences 
approach. Tun and Wingfield (1999) found that speed of information processing predicted listening performance in conditions with one and two competing talkers. Further, Tun et al. (2002) showed a link between a measure of executive control (more specifically, attention-switching control) and recall of words masked by meaningful speech. Furthermore, Humes, Lee, and Coughlin (2006) showed that individual differences in memory capacity were associated with performance differences among older adults in listening situations of divided and selective attention.

The aim of the present study is to investigate, among older adults, whether interference from competing speech can be predicted from individual differences in general inhibitory abilities (i.e., that are not specific to the auditory modality). Given the heterogeneity one normally encounters in groups of older adults, both in terms of auditory and cognitive abilities, the focus here is on individual differences among older adults, rather than on a comparison between age groups. Thus, the results do not directly speak to the debate on whether the inhibitory deficit hypothesis of aging relates to older adults' increased difficulty with competing speech (cf. e.g., Guerreiro et al., 2010; Heifer \& Freyman, 2008; Li, Daneman, Qi, \& Schneider, 2004; Schneider et al., 2000). However, the results will shed more light on how cognitive abilities within an older population may relate to susceptibility to distraction in competing-speech situations.

A variant of the classic Stroop paradigm is used as a measure of inhibitory control, where participants have to name the color of a visually presented rectangle, which either has the letterstring ' $\mathrm{XXXX}^{\prime}$ in it (i.e., the neutral condition) or an incongruent color name (e.g., the word 'GREEN' in a blue rectangle: the incongruent condition). The Stroop color-naming paradigm was chosen, rather than other selective-attention tasks, such as Simon (e.g., Craft \& Simon, 1970) or flanker tasks (Eriksen \& Eriksen, 1974), as the Stroop color-naming paradigm requires participants to ignore competing words, rather than incongruent symbols, letters or spatial information. As such, this variant may be more closely linked to the task of listening in multi-talker conditions. One should note the literature on lowto-moderate correlations among interference scores derived from different versions of the Stroop task (i.e., versions differing in the type of information that has to be ignored: e.g., color names or positional information), which may be problematic for construct validity of any single variant of the Stroop paradigm as a measure of inhibition (e.g., Kramer, Humphrey, Larish, Logan \& Strayer, 1994; Salthouse, Atkinson, \& Berish, 2003; Salthouse \& Meinz, 1995; Schilling, Chetwynd, \& Rabbitt, 2002; Spieler et al., 1996; Ward, Roberts, \& Phillips, 2001).

The present study deviates from prior research with respect to the method to investigate speech processing. Most studies on aging and distraction from competing speech have looked at offline speech comprehension 
(i.e., at which signal-to-noise ratio do listeners achieve $50 \%$ correct identification, or how much do listeners remember from the target talker's speech). By doing so, a number of these studies have provided support for the effortfulness hypothesis (laid out first by Rabbitt, 1968), showing that the extra effort a hearing-impaired listener has to expend to arrive at high accuracy levels comes at the cost of processing resources that would otherwise be available for higher-level processes, such as encoding the speech material in memory (McCoy et al., 2005; Murphy, Craik, Li, \& Schneider, 2000; Tun et al., 2009). The present study, however, focuses on the speech recognition process itself and took an online approach. Effortful listening may not only lead to poorer encoding of the speech material in memory, but also to longer speech processing times. The present study investigated not only the result of the speech recognition process, but also the time that listeners need to come up with a response. Response time is a sensitive measure of speech identification effort, particularly when accuracy is at ceiling level (Nix, Mehta, Dye, \& Cutler, 1993). In the present study, in the no-noise condition, response time analysis may reveal that the more hearing-impaired participants have to expend more effort to achieve a high level of accuracy than the better-hearing. The combination of accuracy and response time measures is taken as an index of speech processing difficulty and of listening effort.

Speech processing was measured using the phoneme monitoring task (cf. Connine \& Titone, 1996 for a description of the paradigm). In this task, participants are asked to press a button as soon as they detect a pre-assigned target phoneme (often word-initial plosives). In principle, a participant could detect the target phoneme without recognizing the carrier word. According to the Race model (Cutler \& Norris, 1979), the phoneme detection response is based on a race between a phonetic code, derived from the acoustic signal, and a phonological code derived from the lexical representation of the word. The decision to report the presence of the target phoneme depends on which code is available first. In the more recent Merge model (Norris, McQueen, \& Cutler, 2000), information from the lexical and prelexical routes is taken into account and merged at the decision stage of phoneme monitoring. However, participants tend to focus on the lexical, rather than the prelexical, route when presented with meaningful materials (i.e., materials that mainly consist of real words or sentences). Thus, target phoneme / $\mathrm{t} /$ is detected faster in, e.g., tomato than in a matched nonword like tofako (Cutler, Mehler, Norris, \& Segui, 1987). Furthermore, phoneme monitoring times are faster when target words are in biasing or high-transitional probability contexts than in more neutral contexts (Foss \& Jenkins, 1973; Morton \& Long, 1976). Evidence that both young and older adults benefit from amount of preceding sentence context in their speed of phoneme monitoring was found in Jesse and Janse (in press). Importantly, there are indications that the task demands do not interfere with processing the sentences for meaning, as task requirements do not 
lead to decrements in comprehension, nor in recall (Brunner \& Pisoni, 1982; Ford et al., 1996; Foss \& Blank, 1980). Thus, despite the fact that listeners are involved in a rather 'unnatural' and explicit task, the phoneme monitoring task taps everyday listening for meaning and, at the same time, provides an online view of auditory sentence processing.

The aim of the present study was to investigate to what extent (agerelated) hearing loss and two measures of cognitive abilities (i.e., inhibitory control and information processing speed) predict listening performance in listening situations with and without competing speech. Information processing speed (as measured with the Digit-Symbol substitution test) was included as it was found to be predictive of listening performance in competing-speech conditions by Tun and Wingfield (1999). Furthermore, processing speed may relate to response speed in the phoneme-monitoring task used here. As said, a variant of the Stroop interference task was included as an index of inhibitory abilities as this measure was expected to predict the impact of competingtalker noise on speech processing performance. No further cognitive abilities (such as memory) were assessed to reduce testing time.

\section{METHOD}

\section{Participants}

A group of 39 older adults ( 15 males and 24 females) participated in this study. They were recruited via a call for participation in an article in a local newspaper and in an information letter for Higher Education for the Elderly students. This educational organization is linked to several universities in the Netherlands and offers academic-level courses for people over 55 years of age. The older adults had a mean age of $72(S D=4.9$, range 65-83) and they were all highly educated.

\section{Measures}

\section{Hearing Sensitivity}

Hearing sensitivity was assessed by pure-tone audiometry (air conduction thresholds) with a portable Maico ST 20 audiometer. The mean audiogram is presented in Figure 1. Mean hearing loss, defined as the mean pure-tone threshold over the frequencies 1,2 , and $4 \mathrm{kHz}$ in their best ear was $29 \mathrm{~dB}$ HL $(S D=13$, range 7-54).

\section{Linguistic Ability}

As a test of their linguistic abilities, a vocabulary test was administered in which participants had to select, out of five options, a synonym for a given word (this test is part of the Groningen Intelligence Test: Luteijn \& van der Ploeg, 1983). Linguistic performance may relate to overall performance on 
FigURE 1. Mean hearing threshold (in dB HL) at different frequencies.

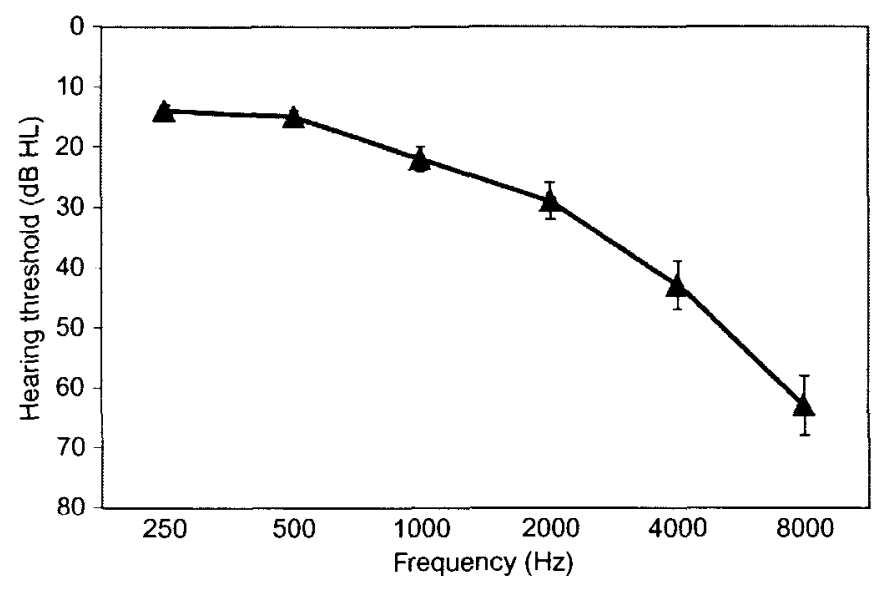

the phoneme monitoring task, but was not expected to specifically predict the impact of competing speech on performance. Mean vocabulary score was 18 out of $20(S D=1.2)$.

\section{Information Processing Speed}

As a measure of information processing speed, participants completed the paper-and-pencil Digit Symbol substitution test, which measures the time needed to recode a number of digits to symbols (cf. also Deary, Johnson, \& Starr, 2010; Hoyer, Stawski, Wasylyshyn, \& Verhaeghen, 2004, Lemke \& Zimprich, 2005). The latter test is part of the Wechsler Adult Intelligence Scale Test (Wechsler, 2004). Mean time needed to code a digit into a symbol was 2.12 seconds $(S D=0.62)$.

\section{Inhibitory Control}

Participants completed a variant of the Stroop color naming test as a measure of effective inhibition of irrelevant stimuli. Pictures were made of rectangles in three colors (blue, green, or red). The width of the rectangles was twice the height. Each rectangle color was combined with two incongruent color names and with a neutral letter string: thus, a blue rectangle would be combined with the words GREEN or RED (in Dutch) in capitals, or with the letter string ' $\mathrm{XXXX}$ '. The pictures were stored in jpeg format. For each rectangle color, the two incongruent presentations were balanced with two occurrences of the same rectangle color with the neutral letter string 
to ensure equal numbers of congruent and incongruent trials in the experiment. These four trials each rectangle color were repeated 10 times, which made up a total of 3 (rectangle colors) $\times 4$ trials $\times 10$ repetitions $=120$ trials (60 incongruent and 60 neutral trials). The order in which the pictures were presented was randomized for each participant. Participants were seated in front of a 17-inch computer screen (distance to screen was about $50 \mathrm{~cm}$ ) on which the colored rectangles were presented centered on the screen. The rectangle picture took up about one-fourth of the screen space. The letters of the color name or letter string were in such a large white font (Arial) that they filled the entire rectangle. The rectangle remained on the computer screen for $1500 \mathrm{~ms}$. Participants had to name the color of the rectangle as fast and as accurately as possible. Then, after a 1000-ms intertrial time during which the screen was blanked, participants proceeded to the next trial. A microphone was placed next to the computer screen which was used to record the participant's responses. The experimental program used for the presentation of the rectangle pictures played a 100 -ms beep, inaudible to the participant, to mark the onset of presentation of each rectangle. Because the beeps were also recorded, the time needed to name the correct color could be determined afterwards for all trials in a speech editing program. Before the actual test started, participants performed a familiarization task with six trials (three incongruent and three neutral trials). Color naming times were analyzed only for the test trials where a correct color response was given. Color naming times were $\log$ transformed to make the data distributions more normal. Mean color naming time (log) in the neutral condition was $2.81(S D=0.05)$. In the incongruent condition, mean color naming time $(\log )$ was $2.90(S D=0.06)$. Before log conversion, mean color naming time was $653 \mathrm{~ms}$ in the neutral condition and $840 \mathrm{~ms}$ in the incongruent condition. Color naming time in the incongruent condition was significantly slower than in the neutral condition $(/(38)=17.03, /$ ? $<.001)$. For each participant, normalized Stroop interference effect was computed (defined as (incongruent-neutral)/neutral). Mean Stroop interference effect was $0.03(S D=0.01)$.

\section{Materials}

Sentence materials were constructed for a phoneme monitoring experiment in which participants were asked to press a response button when they detected a pre-assigned target sound (such as $/ \mathrm{p}, \mathrm{b}, \mathrm{k} /$ ) in a running sentence. There were 76 test sentences all containing the pre-assigned target sound in word-initial position (if $/ \mathrm{p} /$ was the assigned target, the sentence contained, for example, the word pill). The 76 test sentences contained the following target phonemes: /b/ (23 sentences), /d/ (2 sentences), /f/ (1 sentence), /k/ (16 sentences), /p/ (31 sentences), and /v/ (3 sentences). The different 
target phonemes did not occur equally often because the sentence material had not been designed specifically for the purpose of this phoneme monitoring study. The word containing the target sound was not fully predictable from the preceding words in the sentence (e.g., 'I heard that this small pill would improve your digestion'). Additionally, there were 40 filler sentences that did not contain the pre-assigned target sound (also different plosive and fricative sounds for the filler trials), such that participants would not press the button randomly. For practical reasons, the number of filler sentences was relatively low, relative to the number of target sentences. Such a target/filler ratio may lead to a response bias of also responding that the assigned phoneme target is present when it is not actually there. D primes will therefore be reported in the Results section.

The test and filler sentences were spoken by a young male speaker of Standard Dutch. The competing speaker was a male speaker of Standard Dutch as well. Both speakers had been recorded in a sound-insulated booth in the Phonetics laboratory in the Utrecht Institute of Linguistics OTS. The recordings were made with an Audio-Technica AT 8410 microphone on digital audio tape. All sentences were downloaded to a PC, stored as separate files and down sampled to $32 \mathrm{kHz}$. Overall loudness of the audio files was equalized to $72 \mathrm{~dB}$. Each target sentence was presented in a single-talker condition, or in a competing-talker condition, where it was mixed with an equally long sentence of the competing talker. Equal duration of the paired sentences was accomplished by cutting off the competing-talker sentence to the same duration as that of the target-talker's sentence. In the competition condition, the two audio files were mixed for binaural presentation at a target-to-competing talker ratio of $+3.5 \mathrm{~dB}$. Care was taken that the sentence of the competing talker did not contain the target sound for that trial. The two conditions (with and without competing-talker interference) were rotated over the 76 test sentences on two experimental lists, following a Latin square design to avoid multiple presentations of the same sentence. The filler materials (also divided over the conditions) were identical on the two lists, such that each participant was presented with half of the test and half of the filler trials in the nocompetition condition and the other half of the materials in the competition condition.

\section{Procedure}

Participants were seated in front of a computer screen in a soundinsulated booth. The (mixed) auditory material was presented binaurally over closed headphones (Beyer Dynamic DT 250) at a mean level of $80 \mathrm{~dB}$ SPL. Participants were first familiarized with the target talker's voice so that they would know whom of the two competing talkers they would have to attend to. This familiarization phase consisted of the presentation of 10 sentences 
spoken by the target talker. Participants could play these sentences as often as they liked. They were then instructed that each trial would begin with the visual display of the target phoneme for that trial. It was displayed in a large font $1 \mathrm{~s}$ before the auditory presentation started and stayed on the screen during sentence presentation. After sentence offset, participants had one second during which they could still press the button. They would proceed to the next trial after another $1500 \mathrm{~ms}$. The order of the items was randomized for each participant. Participants were told that there would also be filler trials in which the target sound would not occur in the sentence. There were 8 practice trials (4 in the single-talker condition and 4 in the condition with competition) after which participants could still ask questions if the procedure was not clear.

\section{RESULTS}

\section{Intercorrelations Between Measures}

Table 1 provides an overview of intercorrelations between individual characteristics in this participant sample.

Age and hearing loss were not correlated among the older adults. This may be due to the inclusion of three particularly good-hearing adults aged 80 and higher (all three had Pure-Tone-Average thresholds of $25 \mathrm{~dB}$ HL or below). If participants of 80 years and older were excluded, age and hearing loss were significantly correlated $(r=0.52, p=0.001)$. Age correlated with the processing time measure: older participants generally needed more time to code the digits into symbols (cf. Salthouse, 1996). Hearing loss was related to both cognitive measures (i.e., processing speed and inhibitory control): those with more hearing loss generally had longer processing times and showed larger Stroop interference effects. The vocabulary measure correlated with none of the other measures. The outcomes of the two cognitive tests were correlated: processing time was positively related to Stroop effect size

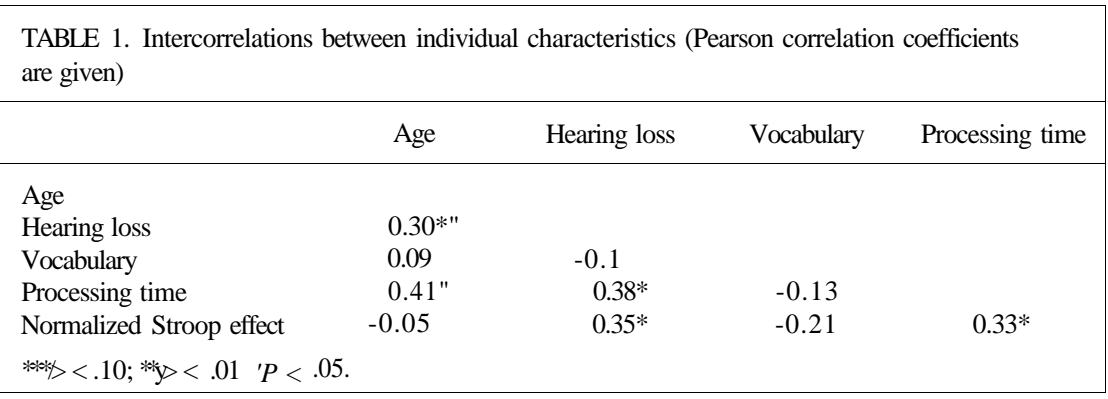


(those with longer processing times showing larger interference effects; cf. Salthouse \& Meinz, 1995).

\section{Phoneme Monitoring Results}

Detection time of the target sound was measured from target sound offset (at the beginning of the first pitch period of the following vowel). From measurements of hit rate and false-alarm rate (participants giving a detection response in filler trials), d primes were computed. Phoneme detection performance (hit rates, false alarm rates, d primes, and mean RTs to targets is given in Table 2.

As a first quick analysis of the data, Mests were carried out on the mean hit rates, $\mathrm{d}$ primes, and mean response times by participants in the two listening conditions. These Mests showed that hit accuracy was significantly lower $(\mathrm{f}(38)=10.6, /$ ? $<.001)$, d prime was significantly lower $(\mathrm{f}(38)=10.5$, $p<.001)$, and that responses were also significantly slower $(?(38)=8.4$, $p<.001)$ in the condition with competition than in the no-competition condition. In order to investigate which background measures are associated with performance in the two listening conditions, one analysis was carried out on the binomial hit data (whether or not one detected the target phoneme) and one on response times. In psycholinguistic designs, multiple crossed random factors (participants and items) affect performance. The more traditional or standard way to analyze psycholinguistic designs would be perform one (by participants only) or two ANOVAs (one with participants as a random effect and one with items as a random effect). Here, mixed-effects modeling was used to analyze the data, as this method allows the researcher to combine two crossed random effects in one analysis and thus to generalize the results across both participants and items (Pinheiro \& Bates, 2000). Tutorial articles such as Quene and van den Bergh (2004, 2008), and Baayen, Davidson, and Bates (2008) show that mixed-effect modeling provides a better way of data analysis than repeated measures ANOVA or univariate analysis of variance when two random factors are involved: joint modeling of the two random factors of participants and items lowers the risk of capitalization on chance (a Type I error). Furthermore, analysis of the hits was done on the binomial

\begin{tabular}{|c|c|c|c|c|}
\hline & Hit rate & False alarm rate & D prime & RT \\
\hline No-noise condition & $94(0.5)$ & $3.2(0.9)$ & $4.1(0.1)$ & $664(9)$ \\
\hline Competing-speech condition & $78(1.6)$ & $9.6(1.3)$ & $2.4(0.1)$ & $782(12)$ \\
\hline
\end{tabular}


data (whether or not a participant had detected the phoneme) rather than on aggregated measures (such as hit or accuracy rates aggregated over participants or items, including d prime) as mixed-effect logistic models are more conservative (also cf. Quene \& van den Bergh, 2008).

Hits

The binomial hit data were analyzed with a mixed-effects version of logistic regression models implemented in the $\mathrm{R}$ statistical program (Version 2.8.0; R Development Core Team, 2007) by using the lmer function of the lme4 library (Bates \& Sarkar, 2009). A binomial logit linking function between responses ( 0 and 1$)$ and predictor variables was included in the models to be able to deal with the categorical nature of the hits. Models evaluated the design variable Condition (with or without competing speech) and the continuous variable Trial number, and the individual background measures as covariates: Age, Gender, Vocabulary test score, Pure-Tone-Average in best ear, Processing speed, and the Stroop effect measure (i.e., the normalized interference effect). Additionally, interactions between Condition and any of the background measures were tested. Whenever two intercorrelated background measures were found to be predictive for performance, the analysis was run again with the measures orthogonalized (for instance, Stroop interference was related to hearing loss, and so the Stroop effect predictor was replaced by the residuals of a linear regression model predicting the Stroop effect as a function of hearing loss).

Models were fit using the residual maximum likelihood criterion. The best-fitting model was established through systematic step-wise model comparisons using likelihood ratio tests. Non-significant effects and interactions were removed from the statistical model predicting the binomial hit data. Age, Gender, Vocabulary score, and Processing speed turned out to be non-significant predictors for hits.

The best-fitting model showed the following effects. For all significant effects and interactions, estimated coefficients $(8 \mathrm{~s})$, with standard errors for 6 in brackets, and p-values are reported. As expected, hit rates were lower in the condition with competition than in the single-talker condition $(\mathrm{B}=$ -2.06 (SE = 0.16), $p<.001)$. Hearing loss did not show a simple effect on performance, but interacted with Condition: the more hearing loss a listener had, the larger the decrement in performance in the competing-speech condition $(\mathrm{B}=-0.03(S E=0.01), p<.05)$. Lastly, the Stroop effect measure was not associated with performance in the single-talker condition, but it was associated with performance in the competing-talker condition $(8=-25.30$ $(S E=9.95), p<.05)$. This is also graphed in Figure 2, showing the relation between the Stroop effect measure and hit rate in the competing-talker condition. The scatter plot shows that the greater the Stroop effect, the lower the hit rate in the competing-talker condition $(r=-.502, p<.01)$. Thus, the 
FIGURE 2. Scatterplot of hit rate in the competing-speech condition against the normalized Stroop interference effect.

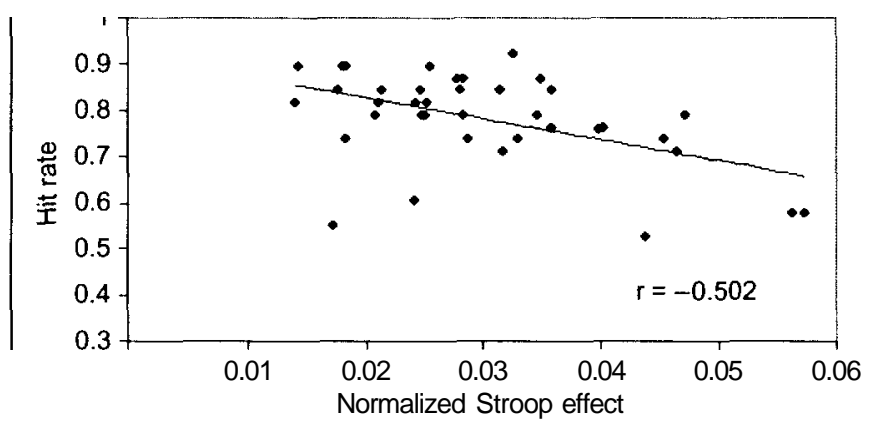

more interference participants experienced in the visual Stroop test, the more interference they experienced from the distractor talker.'

These results indicate that, even after hearing loss and the Stroop effect measure were orthogonalized, the individual Stroop interference measure was significantly associated with speech processing accuracy in the competingspeech condition.

Additional logistic regression models (without any random effects) were run on the data in the competing-speech condition to allow evaluation of the contribution of both predictors (hearing loss and the Stroop effect measure) to proportion of explained variance $\left(R^{2}\right)$. A model only containing Trial number had an $R^{2}$ value of 0 (trial number being insignificant). When hearing loss was added, the $R^{2}$ value increased to 0.029 (yielding a change in $R^{2}$ of .029). After the Stroop effect measure (with hearing loss partialed out) had been added to the model containing Trial number and hearing loss, $R^{2}$ value was increased further to .037 (yielding a change in $R^{2}$ of .008 ). In the latter model (which had a significantly better fit than the previous model only containing hearing loss), both hearing loss $(\beta=-0.024(S E=0.005), p<.001)$ and the Stroop measure $(\beta=-17.48(S E=6.15), p<.01)$ significantly affected accuracy.

\section{Response Times}

Response times (to target phonemes) were analyzed for the entire dataset of correct hit responses. RTs were log-transformed to make the

\footnotetext{
' Simple correlations between individual d prime data (as discussed earlier) and the measures of hearing and of inhibitory show a similar picture. D primes in the competing-speech condition were significantly correlated with hearing loss (Spearman's $r(39)=-.35, p=.029$ ) and with the Stroop effect measure (Spearman's $r(39)=-.33, p=.044)$.
} 
distributions more normal and then tested for main effects and interactions of the same design factors and predictors as in the hit analysis. The log-transformed RTs were analyzed using mixed-effect linear models, as discussed above. Again, age and vocabulary score were non-significant predictors for detection RT. The Stroop effect measure did not predict RTs either, nor did it interact with Condition. Condition significantly affected RTs: detection times were longer in the condition with competition from the distractor talker $(\beta=0.082(0.006), p<.001)$, as was clear from Table 2. Hearing loss was associated with overall RT: the more hearing loss, the slower the response $(\beta=0.005(0.001), p<.001)$. Trial number also affected RT: the later the trial, the slower the response $(\beta=0.0003(0.00008), p<.001)$. The processing speed measure (with hearing loss partialed out) did correlate with RT in the listening experiment: the longer participants took to do the recoding task, the longer their overall phoneme detection time $(\beta=0.16(0.07)$, $p<.05$ ). This relation between individual processing speed and individual mean phoneme detection time is shown in Figure 3.

Summing up, the results support the idea that general inhibitory capacity plays a role in individual susceptibility to distraction from competing speech, but this was only found in the hit rate, and not in the response latency data. Note, however, that RTs become less informative when the accompanying accuracy is lower, if only because there are fewer valid RT data points to analyze. Speed of phoneme monitoring correlated with degree of hearing loss and with general processing speed. Thus, the combination of hit and response time analyses provides a more complete picture than hit rate or RT analysis alone.

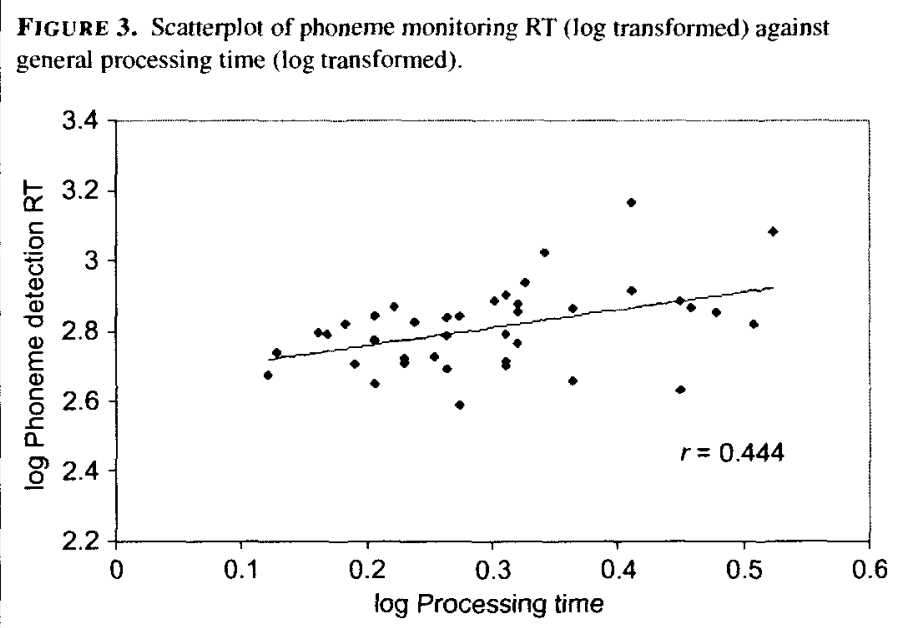




\section{DISCUSSION AND CONCLUSION}

This study was set up to investigate the contribution of age-related hearing loss and general cognitive abilities to older adults' listening difficulty in situations with competing-talker noise. The hypothesis was that interference from a competing talker could be predicted from individual differences in general inhibitory control.

Importantly, in the sample of older adults tested here, hearing loss and the Stroop measure of inhibitory control were correlated, as well as hearing loss and processing speed (cf. Table 1). This is in line with earlier studies showing (strong) correlations between sensory and cognitive function (e.g., Baltes \& Lindenberger, 1997; Lindenberger \& Baltes, 1994).

The present study is the first to show a link between inhibitory control and speech processing performance in multi-talker conditions. As expected, the results indicate that individual differences in speech processing performance in competing-speech situations were primarily associated with hearing sensitivity differences. Inhibitory control was a significant secondary predictor, even with hearing loss partialled out. The present results therefore contribute to earlier literature on links between individual cognitive abilities and susceptibility to distraction in competing-speech conditions (Humes et al., 2006; Tun \& Wingfield, 1999; Tun et al., 2002).

Target detection time in the no-noise condition, as well as in the condition with competing speech, could be predicted by an individual's hearing loss and processing speed. This provides evidence that response time can be taken as a subtle indicator of speech identification effort, particularly in conditions with ceiling accuracy. It remains unclear why the inhibitory-control measure only predicted the impact of competing-talker noise on whether the target phoneme was detected, but not its effect on detection speed. Note that the detection speed analysis showed no interactions between listening condition and any of the background measures. Hearing loss also only predicted overall detection RT, and did not interact with listening condition. As suggested earlier, the absence of such an interaction effect in the RT data may be due to the reduced number of valid data points in the competing-speech condition (in which hit rate ranged between 53 and 92\%).

It is important to note that differences in inhibitory control among young adults may also be associated with differences in listening performance in competing-speech situations. This was not tested in the current study, but is a direction for future investigation.

Cognitive problems (whether age-related or not) are not revealed in standard audiological screening. This makes sense, given that variation in cognitive abilities in normally functioning older adults may generally impact less on speech understanding than hearing impairment. Nevertheless, more knowledge about which cognitive tests are predictive of performance in 
certain communication settings may prove useful with respect to developing more realistic expectations of speech processing performance in difficult communication settings.

Original manuscript received 30 March 2010 Revised manuscript accepted 13 December 2011

First published online 31 January 2012

\section{REFERENCES}

Anderson, N. D., Craik, F. 1. M., \& Naveh-Benjamin, M. (1998). The attentional demands of encoding and retrieval in younger and older adults: 1 . Evidence from divided attention costs. Psychology and Aging, 13, 405-423.

Baayen, R. H., Davidson, D. J., \& Bates, D. M. (2008). Mixed-effects modeling with crossed random effects for subjects and items. Journal of Memory and Language. 59, 390-412.

Baltes, P. B., \& Lindenberger. U. (1997). Emergence of a powerful connection between sensory and cognitive functions across the adult life span: A new window to the study of cognitive aging? Psychology \& Aging, 12, 12-21.

Bates, D. M, \& Sarkar, D. (2009). Ime4: Linear mixed-effects models using s4 classes (Version R package version 0.999375-27).

Brungart, D. S. (2001). Informational and energetic masking effects in the perception of two simultaneous talkers. Journal of the Acoustical Society of America, 109, 1101-1109.

Brunner, H., \& Pisoni, D. B. (1982). Some effects of perceptual load on spoken comprehension. Journal of Verbal Learning and Verbal Behavior, 21, 186-195.

Bugg, J. M., DeLosh, E. L., Davalos, D. B,, \& Davis, H. P. (2007). Age differences in Stroop interference: Contributions of general slowing and task-specific deficits. Aging, Neuropsychology, and Cognition, 14, 155-167.

Connelly, S. L., Hasher, L., \& Zacks, R. T. (1991). Age and reading: The impact of distraction. Psychology and Aging, 6, 533-541.

Connine, C. M, \& Titone, D. (1996). Phoneme monitoring. Language and Cognitive Processes, 11, 635-645.

Craft, J. L., \& Simon, J. R. (1970). Processing symbolic information from a visual display: Interference from an irrelevant cue. Journal of Experimental Psychology, 83, 415^120.

Cutler, A., \& Norris, D. (1979). Monitoring sentence comprehension. In W. E. Cooper \& E. T. C. Walker (Eds.), Sentence processing: Psycholinguistic studies presented to Merrill Garrett (pp. 113-134). Hillsdale, NJ: Lawrence Erlbaum Associates.

Cutler, A., Mehler. J., Norris, D. \& Segui, J. (1987). Phoneme identification and the lexicon. Cognitive Psychology, 19, 141-177.

Deary, I. J., Johnson, W., \& Starr, J. M. (2010). Are processing speed tasks biomarkers of cognitive aging? Psychology and Aging, 25, 219-228.

Dubno, J. R., Dirks, D. D., \& Morgan, D. E. (1984). Effects of age and mild hearing loss on speech recognition in noise. Journal of the Acoustical Society of America, 76. 87-96.

Eriksen, B. A., \& Eriksen, C. W. (1974). Effects of noise letters upon the identification of a target letter in a nonsearch task. Perception \& Psychophysics. 16, 14.3-149.

Ford, J. M,, Woodward, S. H., Sullivan, E. V., Isaacks, B. G., Tinklenberg, J. R,, Yesavage. \& Roth, W. T. (1996). N400 evidence of abnormal responses to speech in Alzheimer's disease. Electroencephalography and Clinical Neurophysiology, 99. 235-246.

Foss, D. J., \& Blank, M. A. (1980). Identifying the speech codes. Cognitive Psychology, 12. $1-31$. 
Foss, D. J., \& Jenkins, C. M. (1973). Some effects of context on the comprehension of ambiguous sentences. Journal of Verbal Learning and Verbal Behavior, 12, 577-589.

Garcia Lecumberri, M. L., \& Cooke, M. P. (2006). Effect of masker type on native and nonnative consonant perception in noise. Journal of the Acoustical Society of America, 119, 2445-2454.

Gordon-Salant, S., \& Fitzgibbons, P. J. (1993). Temporal factors and speech recognition performance in young and elderly listeners. Journal of Speech Language and Hearing Research, $36,1276-1285$.

Guerreiro, M. J. S., Murphy, D. R,, \& van Gerven, P. W. M. (2010). The role of sensory modality in age-related distraction: A critical review and a renewed view. Psychological Bulletin, 756,975-1022.

Hasher, L., \& Zacks, R. T. (1988). Working memory, comprehension, and aging: A review and a new view. Psychology Learning and Motivation, 22, 193-225.

Hasher, L., Zacks, R. T, \& May, C. P. (1998). Inhibitory control, circadian arousal, and age. In D. Gopher \& A. Koriat (Eds.), Attention and performance XVII: Cognitive regulation of performance. Interaction of theory and application (pp. 653-675). Cambridge, MA: MIT Press.

Head, D., Kennedy, K. M., Rodrigue, K. M., \& Raz, N. (2009). Age-differences in perseveration: Cognitive and neuroanatomical mediators of performance on the Wisconsin Card Sorting Test. Neuropsychologia, 47, 1200-1203.

Heifer, K. S., \& Freyman, R. L. (2008). Aging and speech-on-speech masking. Ear \& Hearing, 29, 87-98.

Houx, P. J.. Jolles, J., \& Vreeling, F. W. (1993). Stroop interference: Aging effects assessed with the Stroop Color-Word test. Experimental Aging Research, 19, 209-224.

Hoyer, W. J., Stawski, R. S, Wasylyshyn, C, \& Verhaeghen, P. (2004). Adult age and Digit Symbol Substitution performance: A meta-analysis. Psychology and Aging, 19, 211-214.

Humes, L. E., Lee, J. H., \& Coughlin, M. P. (2006). Auditory measures of selective and divided attention in young and older adults using single-talker competition. Journal of the Acoustical Society of America, 120, 2926-2937.

Jesse, A. \& Janse, E. (in press). Audiovisual benefit for recognition of speech presented with single-talker noise in older listeners. Language and Cognitive Processes.

Kramer, A. F., Humphrey, D. G., Larish, J. F, Logan, G. D., \& Strayer, D. L. (1994). Aging and inhibition: Beyond a unitary view of inhibitory processing in attention. Psychology and Aging, 9, 491-512.

Kwong See, S. T, \& Ryan, E. B. (1995). Cognitive mediation of adult age differences in language performance. Psychology and Aging, 10, 458^168.

Lemke, U., \& Zimprich, D. (2005). Longitudinal changes in memory and processing speed in old age. Aging, Neuropsychology and Cognition, 12, 57-77.

Li, L., Daneman, M., Qi, J. G., \& Schneider, B. A. (2004). Does the information content of an irrelevant source differentially affect spoken word recognition in younger and older adults'? Journal of Experimental Psychology: Human Perception and Performance. 30, 1077-1091.

Lindenberger, U., \& Baltes, P. B. (1994). Sensory functioning and intelligence in old age: A powerful connection. Psychology and Aging, 9, 339-355.

Luteijn, F, \& van der Ploeg, F. A. E. (1983). Handleiding Groninger Intelligentietest (Manual Groningen Intelligence Test). Lisse, The Netherlands: Swets and Zeitlinger.

McCoy, S. L., Tun, P. A., Cox, C. L., Colangelo, M., Stewart, R. A. \& Wingfield, A. (2005). Hearing loss and perceptual effort: Downstream effects on older adults' memory for speech. Quarterly Journal of Experimental Psychology (A), 58, 22-33.

Morton, J.. \& Long, J. (1976). Effect of word transitional probability on phoneme identification. Journal of Verbal Learning and Verbal Behavior, 15, 43-51. 
Murphy, D. R., Craik, F. I. M., Li, K. Z. H., \& Schneider, B. A. (2000). Comparing the effects of aging and background noise on short-term memory performance. Psychology and Aging, 15. 323-334.

Murphy, D. R., McDowd, J. M., \& Wilcox, K. A. (1999). Inhibition and aging: Similarities between younger and older adults as revealed by the processing of unattended auditory information. Psychology and Aging, 14,44-59.

Nix, A. J., Mehta. G., Dye, J., \& Cutler, A. (1993). Phoneme detection as a tool for comparing perception of natural and synthetic speech. Computer, Speech and Language. 7, 211-228.

Norris, D., McQueen, J. M., \& Cutler, A. (2000). Merging information in speech recognition: Feedback is never necessary. Behavioral and Brain Sciences. 23. 299-370.

Phillips, S. L., Gordon-Salant, S., Fitzgibbons, P. J.. \& Yeni-Komshian, G. (2000). Frequency and temporal resolution in elderly listeners with good and poor word recognition. Journal of Speech Language and Hearing Research, 43. 217-228.

Pinheiro. J. C, \& Bates. D. M. (2000). Mixed-Effects Models in S and S-Plus. New York. NY: Springer.

Quene, H.. \& van den Bergh. H. (2004). On multi-level modeling of data from repeated measures designs: A tutorial. Speech Communication, 43. 103-121.

Quene, H., \& van den Bergh. H. (2008). Examples of mixed-effects modeling with crossed random effects and with binomial data. Journal of Memory and Language, 59. 413^125.

Rabbitt, P. M. A. (1968). Channel capacity, intelligibility and immediate memory. Quarterly Journal of Experimental Psychology. 20, 241-248.

Rhodes, M. G. (2004). Age-related differences in performance on the Wisconsin Card Sorting Test: A meta-analytic review. Psychology and Aging, 19, 482^194.

Salthouse, T. A. (1991). Mediation of adult age-differences in cognition by reductions in working memory and speed of processing. Psychological Sciences, 2, 179-183.

Salthouse. T. A. (1996). The processing speed theory of adult age differences in cognition. Psychological Review, 103, 403^128.

Salthouse, T. A., Atkinson, T. M., \& Berish, D. E. (2003). Executive functioning as a potential mediator of age-related cognitive decline in normal adults. Journal of Experimental Psychology. 132. 566-594.

Salthouse, T. A., \& Meinz, E. J. (1995). Aging, inhibition, working memory, and speed. Journals of Gerontology: Series B: Psychological Sciences and Social Sciences, 50, P297-P306.

Schilling, V. M., Chetwynd. A. \& Rabbitt, P. M. A. (2002). Individual inconsistency across measures of inhibition: An investigation of the construct validity of inhibition in older adults. Neuropsychologia, 40, 605-619.

Schneider, B. A.. Daneman. M,, Murphy. D. R.. \& Kwong See. S. (2000). Listening to discourse in distracting settings: The effects of aging. Psychology and Aging. 15, 110-125.

Spieler. D. H., Balota. D. A., \& Faust, M. E. (1996). Stroop performance in healthy younger and older adults and in individuals with dementia of the Alzheimer's type. Journal of Experimental Psychology: Human Perception and Performance, 22, 461^179.

Tun, P. A., McCoy, S.. \& Wingfield, A. (2009). Aging, hearing acuity, and the attentional cost of effortful listening. Psychology and Aging, 24, 761-766.

Tun, P. A., O'Kane, G., \& Wingfield, A. (2002). Distraction by competing speech by young and older adult listeners. Psychology and Aging, 17, 453^167.

Tun, P. A., \& Wingfield, A. (1999). One voice too many: Adult age differences in language processing with different types of distracting sounds. Journal of Gerontology: Psychological Sciences, 54B, P317-P327.

Tremblay, K. L.. Piskosz, M.. \& Souza. P. (2003). Effects of age and age-related hearing loss on the neural representation of speech cues. Clinical Neurophysiology. 114. 1332-1343. 


\section{ESTHER JANSE}

Van Engen, K. J., \& Bradlow, A. R. (2007). Sentence recognition in native and foreignlanguage multi-talker background noise. Journal of the Acoustical Society of America, 121, 519-526.

Ward. G,, Roberts. M. J., \& Phillips, L. H. (2001). Task-switching costs, Stroop-costs, and executive control: A correlational study. Quarterly Journal of Experimental Psychology, 54A, 491-511.

Wechsler, D. (2004). Wechsler Adult Intelligence Test (Dutch version, 3rd ed.). Amsterdam, The Netherlands: Harcourt Test Publishers.

Zacks. R. T. \& Hasher, L. (1994). Directed ignoring: Inhibitory regulation of working memory. In D. Dagenbach \& T. H. Carr (Eds.), Inhibitory processes in attention, memory and language (pp. 241-264). San Diego, CA: Academic Press. 